\title{
LOWER BOUND ON THE DRAG OFFERED TO A NEWTONIAN FLUID SPHERE PLACED IN A FLOWING ELLIS FLUID*
}

\author{
V. MOHAN AND D. VENKATESWARLU \\ Department of Chemical Engineering, Indian Institute of \\ Technology, Madras, India
}

The lower bound on the drag offered to a Newtonian fluid sphere placed in an Ellis model fluid in creeping flow has been found using variational principles. For a solid sphere, the results fall below those reported earlier.

The hydrodynamics of the fall of fluid spheres in quiescent non-Newtonian media has been studied by many investigators in recent years. The creeping flow regime is particularly suited to theoretical analysis in spite of the complexities introduced by the non-Newtonianism. Johnson ${ }^{2)}$ formulated the variational principles for creeping flow of a nonNewtonian fluid. More recently Slattery ${ }^{7)}$ and Yoshioka and Adachi $^{9,10)}$ obtained similar principles using a different approach. These principles have been employed to investigate the flow of a nonNewtonian fluid past a solid sphere ${ }^{1,5,6,8}$. Nakano and Tien $^{3)}$ analyzed the flow of a power-law fluid past a Newtonian fluid sphere and obtained the upper bound on the drag.

The power law model is too simplified a model to represent real fluid behavior. The Ellis model, though mathematically complex, is better descriptive of real fluids and reduces to power law and Newtonian behavior as special cases.

In this paper, the lower bound on the drag offered to a Newtonian fluid sphere placed in an Ellis fluid in creeping flow has been obtained using variational principles.

\section{Analysis}

The equation of continuity and motion can be written in the tensorial form as $^{7)}$

$$
\begin{aligned}
& \frac{\partial \rho}{\partial t}=-\left(\rho v^{i}\right)_{, i} \\
& \rho\left(\frac{\partial v^{i}}{\partial t}+v^{j} v^{i},{ }_{j}\right)=-p^{, i}+\tau^{i j}{ }_{, j}+\rho f^{i}
\end{aligned}
$$

The following assumptions are made:

\footnotetext{
* Received on June 30, 1973

D. Venkateswarlu

Department of Chemical Engineering, Indian Institute of Technology, Madras 600036, India
}

1. The flow is steady, axisymmetric and creeping

2. The fluid particle is perfectly spherical

3. Fluid properties are constant

4. The rheological behaviour of the internal and external fluids are given by

$$
\begin{aligned}
& \tau=2 \eta_{i} \boldsymbol{D} \quad \text { (internal fluid) } \\
& \boldsymbol{\tau}=\frac{2}{\beta} \boldsymbol{D} \quad \text { (external fluid) }
\end{aligned}
$$

where

$$
\beta=\frac{1}{\eta}=\frac{1}{\eta_{0}}\left\{1+\left(\frac{\Pi_{\tau}^{0.5}}{\sqrt{ } 2 \tau_{1 / 2}}\right)^{\alpha-1}\right\}
$$

The equation of motion for the internal fluid reduces to

$$
\begin{aligned}
D^{4} \psi_{i} & =0 \\
D^{4} & =\left\{\frac{\partial^{2}}{\partial r^{2}}+\frac{\sin \theta}{r^{2}} \frac{\partial}{\partial \theta}\left(\frac{1}{\sin \theta} \frac{\partial}{\partial \theta}\right)\right\}^{2}
\end{aligned}
$$

where $\phi_{i}$ is the internal stream function. The velocities are then related to the stream function by

$$
\begin{aligned}
& v_{r}=-\frac{1}{r^{2} \sin \theta} \frac{\partial \psi_{i}}{\partial \theta} \\
& v_{\theta}=\frac{1}{r \sin \theta} \frac{\partial \phi_{i}}{\partial r}
\end{aligned}
$$

The work function $\boldsymbol{E}$, the complimentary work function $E_{c}$ and the function $H_{\tau}$ for the flow field (internal or external) are defined as

$$
\begin{gathered}
\boldsymbol{E}=\int_{0}^{I I} \eta d \Pi \\
E_{c}=\int_{0}^{\Pi_{\tau}} \frac{d \Pi_{\tau}}{4 \eta} \\
H_{\tau}=-\int_{V(s)} E_{c}^{*} d V+\int_{S(s)} \boldsymbol{v} \cdot\left[\tau^{*}-(p+\rho \Phi)^{*} \boldsymbol{I}\right] \cdot \boldsymbol{n} d S
\end{gathered}
$$

where the superscript asterisk denotes quantities obtained from trial stress profiles that satisfy Cauchy's first law and prescribed boundary conditions on the stress tensor. 
It was shown ${ }^{7)}$ that

$$
\int_{V} E d V \geq H_{\tau}
$$

The quantity $\operatorname{tr}(\boldsymbol{\tau} \cdot \boldsymbol{D})$ can be related to $\boldsymbol{E}$. For a Newtonian fluid,

$$
\operatorname{tr}(\boldsymbol{\tau} \cdot \boldsymbol{D})=2 \boldsymbol{E}
$$

and for Ellis fluids with $\alpha \geq 1$,

$$
\operatorname{tr}(\boldsymbol{\tau} \cdot \boldsymbol{D}) \geq \frac{\alpha+1}{\alpha} \boldsymbol{E}
$$

Using Eqs. (10) to (12),

$$
\begin{gathered}
\int_{V_{i}} \operatorname{tr}(\boldsymbol{\tau} \cdot \boldsymbol{D})_{i} d V+\int_{V_{o}} \operatorname{tr}(\boldsymbol{\tau} \cdot \boldsymbol{D})_{o} d V \\
\geq 2 \int_{V_{i}} E_{i} d V+\frac{\alpha+1}{\alpha} \int_{V_{o}} E_{o} d V \\
\geq 2\left(H_{\tau}\right)_{i}+\frac{\alpha+1}{\alpha}\left(H_{\tau}\right)_{o}
\end{gathered}
$$

From a macroscopic energy and momentum balance it can be shown that

$$
V_{\infty} F_{d}=\int_{V_{i}+V_{o}} \operatorname{tr}(\boldsymbol{\tau} \cdot \boldsymbol{D}) d V
$$

The bound on $V_{\infty} F_{d}$ can be obtained by maximising the expression to the right of the inequality (13) subject to the constraints given by the Eq. (5) and the set of boundary conditions

$$
\begin{array}{cc}
\left(v_{r}\right)_{i}=\left(v_{r}\right)_{o}=0 & \text { at } r=1 \\
\left(v_{\theta}\right)_{i}=\left(v_{\theta}\right)_{o} & \text { at } r=1 \\
\left(\tau_{r \theta}\right)_{i}=\left(\tau_{r \theta}\right)_{o} & \text { at } r=1 \\
\left(v_{r}\right)_{i} \text { and }\left(v_{\theta}\right)_{i} & \text { remain finite as } r \rightarrow 0 \\
\tau \rightarrow 0 \text { as } r \rightarrow \infty
\end{array}
$$

It can be seen that the evaluation of $H_{\tau}$ given by Eq. (9) presents a major difficulty. The surface integral has to be calculated over the interface for the inside fluid, and over the interface and a sphere of radius infinity for the external fluid. Though the velocity is specified everywhere on the sphere of radius infinity, the actual velocity at the interface is not known. If the trial functions agree well with the actual field at least at the interface, the velocity at the interface $\boldsymbol{v}$ may be assumed to be $\boldsymbol{v}^{*}$. However, this need not necessarily be so. The surface integral over the interface can be avoided by considering the sum $\left(H_{\tau_{i}}+H_{\tau_{0}}\right)$. Because of Eqs. (15a) to (15c), the integrands in the surface integrals in Eq. (9) are continuous at the interface. Since the normal is radially outward for the fluid shpere, and inward for the external fluid, these integrals cancel in the evaluation of $\left(H_{\tau_{i}}+H_{\tau_{0}}\right)$. Therefore

$$
\begin{aligned}
\left(H_{\tau}\right)_{i}+\left(H_{\tau}\right)_{o}= & -\int_{V_{i+V_{o}}} E_{c}^{*} d V \\
& +\int_{S_{(r=\infty)}} \boldsymbol{v} \cdot\left[\tau^{*}-(p+\rho \Phi)^{*} \boldsymbol{I}\right] \cdot \boldsymbol{n} d S
\end{aligned}
$$

From Eq. (13),

$$
\begin{gathered}
\int_{V_{i}} \operatorname{tr}(\boldsymbol{\tau} \cdot \boldsymbol{D})_{i} d V+\int_{V_{o}} \operatorname{tr}(\boldsymbol{\tau} \cdot \boldsymbol{D})_{o} d V \geq 2 H_{\tau_{i}}+\frac{\alpha+1}{\alpha} H_{\tau_{o}} \\
\geq \frac{\alpha+1}{\alpha}\left(H_{\tau_{i}}+H_{\tau_{o}}\right)=H \text { for } \alpha \geq 1
\end{gathered}
$$

Inequality (17) gives the bound on the lower bound for the total energy dissipation rate. Combining Eqs. (14), (16) and (17),

$$
\begin{aligned}
& V_{\infty} F_{d} \geq \frac{\alpha+1}{\alpha}\left[-\int_{V i+V_{o}} E_{c}^{*} d V\right. \\
& \left.+\int_{S_{(r=\infty)}} \boldsymbol{v} \cdot\left[\tau^{*}-(p+\rho \Phi)^{*} \boldsymbol{I}\right] \cdot \boldsymbol{n} d S\right]=H
\end{aligned}
$$

For evaluating the right side of inequality (18), trial extra-stress functions are assumed for the flow fields. For the internal fluid the stream function is chosen to be of the form

$$
\psi_{1}^{*}=\left(C_{1} r^{2}+C_{2} r^{3}+C_{3} r^{4}\right)\left(1-z^{2}\right) V_{\infty} a^{2}
$$

This is equivalent to assuming the extra stress distributions to be of the form

$$
\begin{aligned}
& \left(\tau_{r \theta}\right)_{i}^{*}=\frac{\eta_{i} V_{\infty}}{a}\left(2 C_{2}+6 C_{3} r\right)\left(1-z^{2}\right)^{1 / 2} \\
& \left(\tau_{\theta \theta}\right)_{i}^{*}=\left(\tau_{\phi \phi}\right)_{i}^{*}=\frac{\eta_{i} V_{\infty}}{a}\left(2 C_{2}+4 C_{3} r\right) z \\
& \left(\tau_{r r}\right)_{i}^{*}=-\frac{\eta_{i} V_{\infty}}{a}\left(4 C_{2}+8 C_{3} r\right) z
\end{aligned}
$$

By the condition $\left(v_{r}\right)_{i}^{*}$ equals zero at $r=1$,

$$
C_{1}+C_{2}+C_{3}=0
$$

Substituting Eq. (19) into Eq. (5),

$$
C_{2}=0
$$

The extra stress distribution in the external fluid is chosen to be $\mathrm{e}^{8)}$

$$
\begin{aligned}
& \frac{\left(\tau_{r \theta}\right)_{o}^{*}}{\eta_{0} V_{\infty} / a}=-A x^{B}\left(1-z^{2}\right)^{1 / 2} \\
& \frac{\left(\tau_{r r}\right)_{o}^{*}}{\eta_{0} V_{\infty} / a}=-\left(C x^{D}+C^{\prime} x^{B}\right) z \\
& \frac{\left(\tau_{\theta \theta}\right)_{o}^{*}}{\eta_{0} V_{\infty} / a}=-\left(F x^{D}+F^{\prime} x^{B}\right) z \\
& \frac{\left(\tau_{\phi \phi}\right)_{o}^{*}}{\eta_{0} V_{\infty} / a}=-\left(E x^{D}+E^{\prime} x^{B}\right) z
\end{aligned}
$$

It can be shown by substituting the extra stress distribution into the equation of motion and equating

$$
\text { that } \begin{aligned}
\frac{\partial^{2}(p+\rho \Phi)_{0}^{*}}{\partial x \partial \theta} & =\frac{\partial^{2}(p+\rho \Phi)_{o}^{*}}{\partial \theta \partial x} \\
E & =F \\
E^{\prime} & =F^{\prime} \\
D & =2 \\
C^{\prime} & =(B-1) A+F^{\prime}
\end{aligned}
$$

It is arbitrarily assumed that $B=4$, its value for the Newtonian case. The boundary condition Eq. (15c) 
reduces to

$$
A=6 C_{1} X
$$

where

$$
X=\eta_{i} / \eta_{o}
$$

The bound on the lower bound on $V_{\infty} F_{d}$ can now be obtained by maximizing $H$ given by Eq. (18) subject to the equality constraints given by Eq. (23), (24) and (30) through (34). Four of the twelve constants appearing in the trial functions are to be chosen to maximize $H$.

\section{Evaluation of $\boldsymbol{H}_{\tau_{i}}+\boldsymbol{H} \tau_{o}$}

Using Eqs. (8) and (20) through (24), it can be shown that, for the internal fluid

$$
-\int_{V_{i}} E_{c}^{*} d V=-16 C_{1}^{2} \pi \eta_{i} V_{\infty}^{2} a
$$

For the external fluid, Eqs. (8), (25) through (28) and (30) through (34) can be combined to yield

$$
\begin{aligned}
- & \int_{V_{o}} E_{c}^{*} d V=-\frac{1}{2} \pi \eta_{o} V_{\infty}^{2} a \int_{-1}^{1} \int_{0}^{1} \\
& \left\{1+2\left(N_{1} \bar{\Pi}_{\tau}^{* 0.5}\right)^{(\alpha-1)} /(\alpha+1)\right\} \bar{\Pi}_{\tau}^{*} x^{-4} d x d z
\end{aligned}
$$

where

$$
\begin{aligned}
\bar{\Pi}_{\tau}^{*}= & x^{8}\left\{2 A^{2}\left(1-z^{2}\right)+z^{2}\left(C^{2}+2 F^{\prime 2}\right)\right\} \\
& +x^{6} z^{2}\left(2 C C^{\prime}+4 F F^{\prime}\right)+x^{4} z^{2}\left(C^{2}+2 F^{2}\right)
\end{aligned}
$$

Substituting the trial extra-stress function in the equation of motion, the trial pressure distribution in the external fluid is obtained as

$$
\frac{(p+\rho \Phi)_{0}^{*}}{\eta_{0} V_{\infty} / a}=-z\left\{\left(A+F^{\prime}\right) x^{4}+F x^{2}\right\}
$$

The surface integral in Eq. (18) can now be evaluated. Making use of the fact that on the sphere of radius infinity the velocity is that of the undisturbed fluid and that the normal is radially outward, it can be shown that

$$
\int_{S_{(r=\infty)}} \boldsymbol{v} \cdot\left[\tau^{*}-(p+\rho \Phi)^{*} \boldsymbol{I}\right] \cdot \boldsymbol{n} d S=\frac{4}{3}(F-C) \pi \eta_{0} V_{\infty}^{2} a
$$

Combining Eqs. (18), (36), (37) and (40),

$$
V_{\infty} F_{d} \geq \frac{\alpha+1}{\alpha}\left[\frac{4}{3}(F-C)-16 C_{1}^{2} X-R / 2\right] \pi \eta_{0} V_{\infty}^{2} a
$$

where

$$
R=\int_{-1}^{1} \int_{0}^{1}\left\{1+2\left(N_{1} \bar{\Pi}_{\tau}^{* 0.5}\right)^{(\alpha-1)} /(\alpha+1)\right\} \Pi_{\tau}^{*} x^{-4} d x d z
$$

The drag coefficient $C_{d}$ and the Reynolds number $R e$ are defined as

$$
\begin{aligned}
C_{d} & =\frac{2 F_{d}}{\pi a^{2} \rho V_{\infty}^{2}} \\
R e & =\frac{2 a V_{\infty} \rho}{\eta_{0}}
\end{aligned}
$$

Equation (41) is reduced to

$$
Y=\frac{C_{d} R e}{24} \geq \frac{\alpha+1}{6 \alpha}\left\{\frac{4}{3}(F-C)-16 C_{1}^{2} X-\frac{R}{2}\right\}
$$

where $R$ is defined by Eq. (42).

\section{Solution}

A numerical technique is employed to evaluate the maxima of the expression on the right of the inequality (45). A search is made on the four variables $C_{1}, C, F$ and $F^{\prime}$ using the method of Rosenbrock ${ }^{4)}$. The maxima so obtained give the lower bound on $Y$. Values of the lower bound $Y_{L B}$ are obtained to a convergence of $10^{-5}$.

\section{Analytical Solution}

For the following two limiting cases, the maximum of the right side of inequality (45) and the constants $C_{1}, C, F$ and $F^{\prime}$ can be obtained analytically.

(i) $N_{1}=0$

and

$$
\begin{aligned}
C_{1} & =1 /(4 X+1) \\
C & =-(3 X+2) /(X+1) \\
F & =-C / 2
\end{aligned}
$$

(ii) $\alpha=1, N_{1} \neq 0$

$$
Y_{L B}=\left(\frac{2+3 X}{3+3 X}\right)\left(\frac{\alpha+1}{2 \alpha}\right)
$$

and

$$
\begin{aligned}
& C_{1}=1 /\{4(2 X+1)\} \\
& C=-(3 X+1) /(2 X+1) \\
& F=-C / 2 \\
& F^{\prime}=-6 C_{1} X \\
& Y_{L B}=(1+3 X) /(3+6 X)
\end{aligned}
$$

The values of $C_{1}, C, F$ and $F^{\prime}$ given by Eq. (48) are used to start the Rosenbrock search for the nonNewtonian case.

\section{Discussion}

Figure 1 is a plot of the variation of $Y_{L B}$ with the viscosity ratio $X$, and indicates that $10^{-2} \leq X \leq 10^{3}$ is a sufficient range for theoretical investigation. This range corresponds to the variation of the fluid particle behavior from that of a bubble to that of a solid sphere.

In Fig. 2 is plotted the variation of the lower bound with the Ellis parameter $\alpha$. The results of Hopke and Slattery ${ }^{1)}$ for a solid sphere fall above those of the present investigation for a viscosity ratio of $10^{3}$. However, since the trial stress function chosen by Hopke and Slattery is a particularisation of that assumed in the present work, their results should have been lower.

Figures 3 and 4 show the effect of the Ellis parameters $N_{1}$ and $\alpha$ on the bound. Four limiting cases arise. 


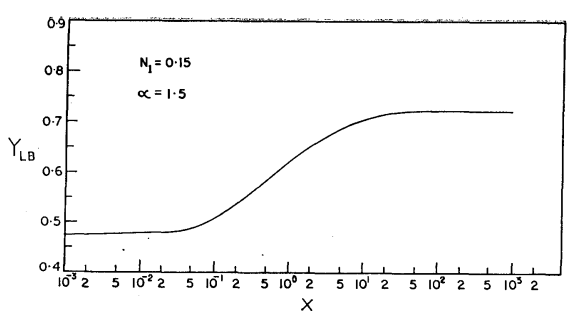

Fig. 1 Lower bound on drag versus viscosity ratio

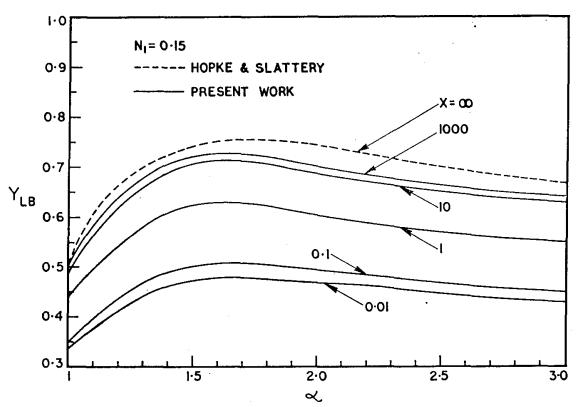

Fig. 2 Lower bound on drag versus $\alpha$ for various values of $X$

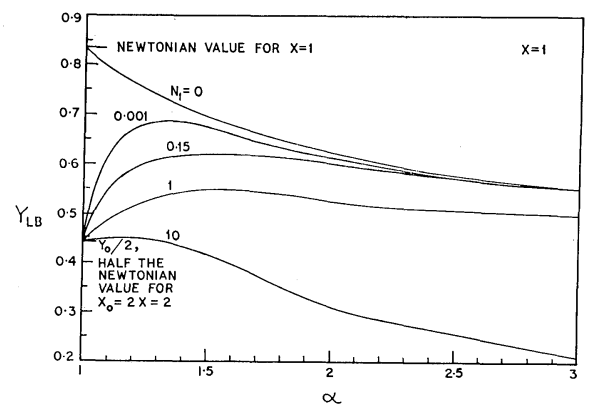

Fig. 3 Effect of Ellis parameters $N_{1}$ and $\alpha$ on the lower bound

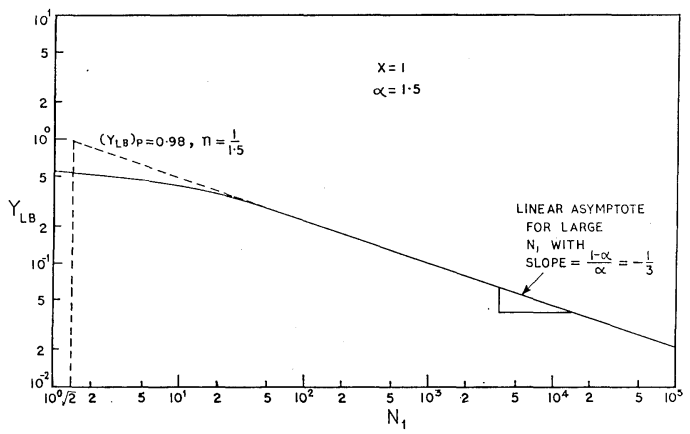

Fig. 4 Behavior of the lower bound for large values of $N_{1}$

1) $N_{1} \rightarrow 0, \alpha \neq 1$.

The bound approaches that given by Eq. (47). Though for $N_{1}=0$ the fluid behavior is Newtonian, the bound for $\alpha \neq 1$ does not correspond to the Newtonian value (Fig. 3). This is because a boundon-bound is obtained for $\alpha \neq 1$, and this value is less than the bound on $Y$.

2) $N_{1} \rightarrow 0, \alpha \rightarrow 1$.

The bound given by Eq. (47) tends to the Newtonian value $(2+3 X) /(3+3 X)$ as $\alpha \rightarrow 1$. The bound for a Newtonian fluid is obtained by taking the limit as $N_{1} \rightarrow 0$ and then taking the limit as $\alpha \rightarrow 1$ (Fig. 3).

3) $N_{1} \neq 0, \alpha=1$.

Though $\alpha=1$ represents a Newtonian fluid, the fluid viscosity given by Eq. (4) is $\eta_{0} / 2$. For this fluid, the viscosity ratio and the Reynolds number are given by

$$
X_{o}=\eta_{i} /\left(\eta_{o} / 2\right)=2 X
$$

and

$$
R e_{0}=2 a V_{\infty} \rho /\left(\eta_{0} / 2\right)=2 R e
$$

Therefore,

$$
Y_{o}=C_{d} R e_{o} / 24=2\left(C_{d} R e / 24\right)=2 Y
$$

This limiting case gives $Y=Y_{o} / 2$ where $Y_{o}$ is the value of $Y$ for a Newtonian fluid with $X_{0}=2 X$. For the specific case $X=1$ (or $\left.X_{o}=2\right), \quad Y_{o}=\left(2+3 X_{o}\right) /$ $\left(3+3 X_{0}\right)=8 / 9$. The limiting value shown in Fig. 3 indicates that $Y_{\alpha=1}=Y_{o} / 2=4 / 9$.

4) $N_{1} \rightarrow \infty$.

It can be shown that for large $N_{1}$, the fluid behaves as a power-law fluid with

$$
\eta=K(2 \Pi)^{(n-1) / 2}
$$

where $n=1 / \alpha$ and $K=\eta_{0}\left(2 N_{1}^{2}\right)^{(1-\alpha) / 2 \alpha}\left(V_{\infty} / a\right)^{(1-\alpha) / \alpha}$

Further, the bound on $Y$ can be related by

$$
Y_{L B}=\left(Y_{L B}\right)_{p}\left(N_{1} / \sqrt{2}\right)^{(1-\alpha) / \alpha}
$$

Equation (53) suggests that a log-log plot of $Y_{L B}$ versus $N_{1}$ is linear for large $N_{1}$ with a slope $(1-\alpha) / \alpha$. This linear asymptote is shown in Fig. 4. It passes through the point $\left(\sqrt{2},\left(Y_{L B}\right)_{p}\right)$ which gives the lower bound on $Y$ for the power-law fluid flow past a solid sphere.

\section{Conclusions}

1) The lower bound on the drag offered by a fluid sphere placed in an Ellis fluid in creeping flow has been found for the first time.

2) For $\alpha \rightarrow 1$, Newtonian behavior is observed. For $N_{1} \rightarrow \infty$, power-law behavior is approached.

\section{Acknowledgment}

One of the authors (V.M.) wishes to acknowledge the financial assistance provided by the Indian Institute of Technology, Madras, and the Council of Scientific and Industrial Research, India.

\section{Nomenclature}

$a \quad=$ radius of the spherical particle $\quad[\mathrm{cm}]$

$A, B, C, C^{\prime}=$ constants in Eq. (25) through (28) [-]

$C_{1}, C_{2}, C_{3}=$ constants in Eq. (19) [-]

$C_{d} \quad=$ drag coefficient $\quad[-]$

$D \quad=$ constant in Eqs. (26) through (28) [-]

D $\quad$ rate-of-deformation tensor [1/sec]

$E, E^{\prime} \quad=$ constants in Eq. (28) [-]

$\boldsymbol{E} \quad=$ work function defined in Eq. (7) [erg/sec]

$\begin{aligned} E_{c}= & \text { complementary work function defined } \\ & \text { in Eq. (8) } \quad[\mathrm{erg} / \mathrm{sec}]\end{aligned}$

$f_{i} \quad=$ body force $\quad\left[\mathrm{cm} / \mathrm{sec}^{2}\right]$ 


\begin{tabular}{|c|c|c|c|c|c|}
\hline$F, F^{\prime}$ & $=$ constants in Eq. $(27)$ & {$[\mathrm{erg} / \mathrm{sec}]$} & $\tau_{1 / 2}$ & $=$ Ellis parameter & [dyne $/ \mathrm{cm}^{2}$ ] \\
\hline$F_{d}$ & $=$ drag force on the sphere & [dyne] & $\phi$ & $=\phi$ co-ordinate & {$[-]$} \\
\hline$H$ & $=$ defined in Eq. (18) & {$[\mathrm{erg} / \mathrm{sec}]$} & $\Phi$ & $=$ potential expressing the body force & {$\left[\mathrm{cm}^{2} / \mathrm{sec}^{2}\right]$} \\
\hline$H_{\tau}$ & $=$ defined in Eq. (9) & {$[\mathrm{erg} / \mathrm{sec}]$} & $\phi$ & $=$ stream function & {$\left[\mathrm{cm}^{3} / \mathrm{sec}\right]$} \\
\hline$I$ & $=$ unit tensor & [-] & $\Pi$ & $=$ second invariant of the rate-of- & \\
\hline$K$ & $=$ consistency index & $\left.\cdot \mathrm{sec}^{n} / \mathrm{cm}^{2}\right]$ & & deformation tensor, $D_{i j} D^{i j}$ & {$\left[1 / \mathrm{sec}^{2}\right]$} \\
\hline$n$ & $=$ flow behaviour index & {$[-]$} & $\Pi_{\tau}$ & $=$ second invariant of the extra-stress & \\
\hline$n$ & $=$ normal vector on the bounding surface & {$[-]$} & & tensor, $\tau_{i j} \tau^{i j}$ & [dyne $\left.{ }^{2} / \mathrm{cm}^{4}\right]$ \\
\hline$N_{1}$ & $=$ Ellis parameter $\left(\eta_{0} V_{\infty}\right) /\left(\sqrt{2} a \tau_{1 / 2}\right)$ & {$[-]$} & $\bar{\Pi}_{\tau}^{*}$ & $=\Pi_{\tau}^{*} /\left(\eta_{0} V_{\infty} / a\right)^{2}$ & {$[-]$} \\
\hline$p$ & $=$ pressure & [dyne $\left./ \mathrm{cm}^{2}\right]$ & \multirow{2}{*}{\multicolumn{3}{|c|}{$\langle$ Subscripts $\rangle$}} \\
\hline$r$ & $=$ dimensionless radius & {$[-]$} & & & \\
\hline$R$ & $=$ defined in Eq. (42) & {$[-]$} & $i$ & $=$ internal fluid & \\
\hline$R e$ & $=$ Reynolds number & {$[-]$} & $o$ & $=$ external fluid & \\
\hline$S(s)$ & $=$ surface bounding the flow system & {$[-]$} & \multicolumn{3}{|c|}{$\langle$ Superscript $\rangle$} \\
\hline$t$ & $=$ time co-ordinate & [sec] & $*$ & $=$ quantities obtained from trial profile & \\
\hline $\boldsymbol{v}$ & $=$ velocity vector & {$[\mathrm{cm} / \mathrm{sec}]$} & \multirow{2}{*}{\multicolumn{3}{|c|}{ Literature Cited }} \\
\hline$V(s)$ & $=$ volume domain of the flow system & {$[-]$} & & & \\
\hline$V_{\infty}$ & $=$ terminal velocity & {$[\mathrm{cm} / \mathrm{sec}]$} & \multirow{5}{*}{\multicolumn{3}{|c|}{$\begin{array}{l}\text { 1) Hopke, S. W. and J. C. Slattery: AIChE J., 16, } 224 \text { (1970) } \\
\text { 2) Johnson, M. W., Jr.: Phys. Fluids, 3, } 871 \text { (1960) } \\
\text { 3) Nakano, Y. and Chi Tien: AIChE J., 14, } 145 \text { (1968) } \\
\text { 4) Rosenbrock, H. H. and C. Storey: “Computational Tech- } \\
\text { niques for Chemical Engineers", p. 64, Pergamon Press } \\
\text { (1966) }\end{array}$}} \\
\hline$X$ & $=\eta_{i} / \eta_{o}$ & {$[-]$} & & & \\
\hline$X_{o}$ & $=$ value of $X$ for $\alpha=1, N_{1} \neq 0$ & {$[-]$} & & & \\
\hline$Y$ & $=C_{d} R e / 24$ & {$[-]$} & & & \\
\hline$Y_{o}$ & $\begin{aligned}= & \text { value of } Y \text { for a Newtonian fluid with } \\
& \text { viscosity ratio } X_{o}\end{aligned}$ & {$[-]$} & & & \\
\hline$Y_{L B}$ & $=$ lower bound on $Y$ & {$[-]$} & \multicolumn{3}{|c|}{ 5) Slattery, J. C.: Appl. Sci. Res., 10A 286 (1961) } \\
\hline$\left(Y_{L B}\right)_{p}$ & $=$ lower bound on $Y$ for a power-law fluid & {$[-]$} & \multicolumn{3}{|c|}{ 6) Slattery, J. C.: AIChE J., 8, 663 (1962) } \\
\hline$\alpha$ & $=$ Ellis parameter & {$[-]$} & \multicolumn{3}{|c|}{$\begin{array}{l}\text { 7) Slattery, J. C.: "Momentum, Energy and Mass Transfer in } \\
\text { Continua", McGraw Hill (1972) }\end{array}$} \\
\hline$\beta$ & $=$ fluidity in Eq. (3b) & [1/poise $]$ & \multirow{2}{*}{\multicolumn{3}{|c|}{$\begin{array}{l}\text { 8) Wasserman, M. L. and J. C. Slattery: AIChE J., 10, } 383 \\
\text { (1964) }\end{array}$}} \\
\hline$\eta$ & $=$ viscosity & [poise] & & & \\
\hline$\theta$ & $=\theta$ co-ordinate & {$[-]$} & \multirow{2}{*}{\multicolumn{3}{|c|}{$\begin{array}{l}\text { 9) Yoshioka, N. and K. Adachi: J. Chem. Eng. Japan., 4, } 217 \\
\text { (1971) }\end{array}$}} \\
\hline$\rho$ & $=$ density & {$\left[\mathrm{gm} / \mathrm{cm}^{3}\right]$} & & & \\
\hline$\tau$ & $=$ extra stress tensor & {$\left[\right.$ dyne $\left./ \mathrm{cm}^{2}\right]$} & \multicolumn{3}{|c|}{ 10) Yoshioka, N. and K. Adachi: ibid., 4, 221 (1971) } \\
\hline$\tau_{i j}$ & $=$ components of the extra stress tensor & {$\left[\right.$ dyne $\left./ \mathrm{cm}^{2}\right]$} & & & \\
\hline
\end{tabular}

\title{
STUDIES OF FLUIDIZATION OF MOIST PARTICLES*
}

\author{
Norio ARAI AND SACHIo SUGIYAMA \\ Department of Chemical Engineering, Nagoya University, Nagoya
}

In this paper, the effect of moisture content on the quality of fluidization in the gas-solid fluidized bed was investigated experimentally and theoretically, using nonporous particles such as glass bead, glass powder, silica sand and so on. It was confirmed that the minimum fluidizing velocity $\left(u_{\mathrm{m} f}\right)$ of moist particles can be estimated from existing theory with aid of the observed value of the minimum fluidizing voidage $\left(\varepsilon_{\mathrm{mf}}\right)$, provided the moisture content (w) is lower than $0.1 \mathrm{wt} \%$.

Further, the expansion ratio of the bed proved well estimable on the lines of Ergun's idea except in the case of high expansion ratio.

\section{Introduction}

The operations of drying, granulation and removal of mists from gases with a bed of fluidized solids have

\footnotetext{
* Received on December 10, 1973

Presented at the 7th Autumn Meeting of The Soc. of Chem.

Engrs., Japan (October 14, 1973)

干464 名古屋市千種区不老町

名古屋大工学部化学工学科 杉山幸男
}

been studied ${ }^{5,7)}$. Since the behavior of fluidization is considerably influenced by the presence of moisture, fluidization becomes impossible under certain circumstances. A few reports ${ }^{1,6,9)}$ have been presented on the fluidization of moist particles. However, the effect of moisture content on the behavior of fluidization seems to have not yet been investigated in detail, considering physical properties of the 